\section{Análise da implantação do sistema de atendimento pré-hospitalar móvel em cinco capitais brasileiras}

\author{
Analysis of the implementation of a mobile \\ pre-hospital treatment system in five Brazilian \\ state capitals
}

\author{
${ }_{1}$ Escola Nacional de Saúde \\ Pública Sergio Arouca, \\ Fundação Oswaldo Cruz, \\ Rio de Janeiro, Brasil. \\ 2 Instituto Fernandes \\ Figueira, Fundação Oswaldo \\ Cruz, Rio de Janeiro, Brasil. \\ Correspondência \\ M. C. S. Minayo \\ Centro Latino-Americano de \\ Estudos de Violência e Saúd \\ Jorge Careli, Escola Nacional \\ de Saúde Pública Sergio \\ Arouca, Fundação \\ Oswaldo Cruz. \\ Av. Brasil 4036, sala 700 \\ Rio de Janeiro, $R J$ \\ 21040-361, Brasil. \\ cecilia@claves.fiocruz.br
}

\begin{abstract}
The article presents a description and analysis of the implementation of a pre-hospital treatment system (SAMU) as part of the research project Diagnostic Analysis of the Implementation of a National Policy for the Reduction of Violence and Accidents. Implementation and organization of the SAMU service, together with the related materials, human resources, and equipment, was studied in five Brazilian State capitals with high morbidity and mortality rates from external causes: Curitiba (Paraná), Recife (Pernambuco), Brasília (Federal District), Rio de Janeiro, and Manaus (Amazonas). The study involved four phases, each developing exploratory and analytical cycles, combined with fieldwork, triangulating quantitative and qualitative data. Implementation of the pre-hospital treatment system is now a key health sector asset. Further necessary steps include: comprehensive legislation covering vehicles, personnel, and equipment; closer networking between mobile units and healthcare facilities; focus on information generated in this sub-system, thus facilitating planning; and maintaining and upgrading high qualifications for SAMU crews. The service is officially establishing, standardizing, and regulating a sub-system that is crucial for saving lives.
\end{abstract}

Prehospital Care; Emergencies; Prehospital Services
Maria Cecília de Souza Minayo ${ }^{1}$

Suely Ferreira Deslandes 2

\section{Introdução}

Este artigo apresenta descrição e análise da implantação e da implementação de um sistema de atendimento pré-hospitalar móvel (Serviço de Atendimento Móvel de Urgência - SAMU), oficializado pelo Ministério da Saúde por meio do Decreto $n^{\circ}$. 5.055, de 27 de abril de $2004{ }^{1}$. Esse decreto foi acompanhado por várias portarias, citadas neste artigo, que o regulam de acordo com as diretrizes da Política Nacional de Redução da Morbimortalidade por Acidentes e Violências 2. Os dados primários aqui descritos e analisados fazem parte de um estudo empírico denominado Análise Diagnóstica sobre Implantação da Política Nacional de Redução de Acidentes e Violências, realizado por pesquisadores do Centro Latino Americano de Estudos sobre Violência e Saúde, Escola Nacional de Saúde Pública Sergio Arouca, Fundação Oswaldo Cruz (CLAVES/ENSP/FIOCRUZ) 3 em cinco capitais brasileiras onde violências e acidentes constituem relevantes causas de traumas e de mortes. O estudo completo analisou os subsistemas préhospitalar, hospitalar e de reabilitação, bem como ações de prevenção e promoção da vida, em Brasília (Distrito Federal), Curitiba (Paraná), Manaus (Amazonas), Recife (Pernambuco) e Rio de Janeiro. Portanto, o objeto deste artigo é um recorte do referido estudo.

Considera-se atendimento pré-hospitalar toda e qualquer assistência realizada, direta ou indiretamente, fora do âmbito hospitalar, utili- 
zando-se meios e métodos disponíveis. Esse tipo de atendimento pode variar de um simples conselho ou orientação médica até o envio de uma viatura de suporte básico ou avançado ao local da ocorrência onde haja pessoas traumatizadas, visando à manutenção da vida e à minimização de seqüelas. No Brasil, o sistema se divide em serviços móveis e fixos. O pré-hospitalar móvel, objeto desta reflexão, tem como missão o socorro imediato das vítimas que são encaminhadas para o atendimento pré-hospitalar fixo ou para o atendimento hospitalar 4 .

$\mathrm{O}$ atendimento pré-hospitalar, seja móvel, seja fixo, tem como premissa o fato de que, dependendo do suporte imediato oferecido à vítima, lesões e traumas podem ser tratados sem gerar seqüelas significativas.

Um dos fatores críticos que interfere no prognóstico das vítimas de trauma é o tempo gasto até que o tratamento definitivo possa ser efetivado. O Committee on Trauma of American College of Surgeons 5, dos Estados Unidos, estabelece o tempo de vinte minutos como intervalo máximo ideal para execução dos primeiros procedimentos, em casos graves 6,7. A necessidade de presteza do atendimento se deve ao fato de que as primeiras horas pós-evento traumático têm sido apontadas por vários autores 7,8 como o período de maior índice de mortalidade. Estudos de Champion et al. ${ }^{9}$ constatam que, em geral, entre as vítimas de traumas, mais da metade não chega a resistir 24 horas.

Albuquerque \& Minayo 10 e Deslandes 11 indicam que o modelo pré-hospitalar móvel vigente em quase todas as partes da sociedade ocidental tem sido inspirado na organização de origem americana e francesa. A primeira prioriza o atendimento feito por paramédicos (técnicos), enquanto a segunda adota a presença de médicos nas ambulâncias 12 .

Os denominados paramédicos, de acordo com a legislação de cada estado americano, podem realizar a administração de medicamentos. No Japão e na Inglaterra, por exemplo, esses profissionais estão aptos também a realizar procedimentos de suporte avançado de vida, tais como desfibrilação, entubação endotraqueal e aplicação de medicamentos por via intravenosa 13,14. Na França, o atendimento é feito por médicos nas unidades móveis, assim os bombeiros se ocupam do resgate de vítimas com lesões de baixa gravidade bem como realizam manobras para a liberação da vítima no caso de ela estar presa a ferragens 15 .

O Brasil oficialmente adotou o modelo francês, o SAMU, adequando-o às peculiaridades nacionais. Seus princípios são: (1) considerar o auxílio médico de urgência uma atividade sanitária;
(2) atuar rapidamente no local do sinistro com procedimentos eficazes e adequados; (3) abordar cada caso com cuidados médicos, operacionais e humanitários; (4) trabalhar em interação nas operações de socorro, mas com responsabilidades estabelecidas para cada profissional; (5) realizar ações preventivas em complementação com a ação de urgência.

O SAMU, no Brasil, propõe um modelo de assistência padronizado que opera com uma central de regulação, com discagem telefônica gratuita e de fácil acesso (linha 192), com regulação médica regionalizada, hierarquizada e descentralizada. Nesse sistema, há uma normalização para a composição das equipes de socorro, segundo complexidade, regulando os tipos de unidades móveis e suas atribuições e recursos. Há protocolos para atendimento de múltiplas vítimas, além de ferramentas operacionais regulares (mapa de área de atuação identificando os pontos de apoio das unidades básicas e das unidades de maior complexidade; grade de referência e contra-referência dos serviços interligados de urgência do município ou região; lista de todos os telefones; mapas para capacidade instalada dos serviços de urgência e viária e mapas de risco) 4.

O período em que a presente pesquisa foi realizada (2003-2006) demarca o início da implantação do SAMU. Esse processo se configura como uma transição do modelo de atendimento realizado tradicionalmente pelo Corpo de Bombeiros ou, em poucos casos, associado com sistemas locais pré-existentes. A transição tem se caracterizado pela convivência dos dois modelos. Historicamente, o Corpo de Bombeiros tem atuado em todo o país, com atendimento em linha própria (193), sem ter necessariamente uma regulação médica. $\mathrm{O}$ atendimento é feito, habitualmente, por técnicos de enfermagem. Em algumas capitais, como é o caso do Rio de Janeiro, contudo, os serviços de bombeiros incluem a presença de médicos, sendo organizados por meio de uma central de regulação e dispondo de ambulâncias diferenciadas, conforme a complexidade.

Este artigo busca dar ênfase aos dados do estudo avaliativo, segundo diretrizes da Política Nacional de Redução da Morbimortalidade por Acidentes e Violências, pesquisa que foi feita com o intuito de contribuir para o monitoramento das ações visando a responder às necessidades da população brasileira.

\section{Metodologia}

O conceito metodológico básico do estudo é o de análise diagnóstica, pautada nas perspectivas compreensivas e interpretativas, que busca dar 
conta: (1) dos êxitos e das dificuldades do sistema de saúde para atender à crescente demanda e às mudanças qualitativas das lesões e traumas provenientes das violências e acidentes; (2) de criar alternativas e possibilidades para o melhor desenvolvimento da gestão. Uma análise situacional leva em conta as condições gerais de infra-estrutura, de pessoal qualificado, de planejamento e de apoio oferecido para o desempenho das ações.

Do ponto de vista operacional, foram adotados os princípios da triangulação de métodos, proposta de articulação interpretativa que integra múltiplos pontos de vista de autores, técnicas, estratégias e métodos 16,17,18.

A investigação que dá origem ao artigo se fez de forma cooperativa entre o CLAVES e vários centros de pesquisa das capitais incluídas: o Departamento de Serviço Social da Universidade de Brasília (Brasília, Distrito Federal); o Núcleo de Estudos em Saúde Coletiva da Universidade Federal do Rio de Janeiro (Rio de Janeiro); a Faculdade de Educação da Universidade Federal de Manaus (Manaus, Amazonas); a Pontifícia Universidade Católica de Curitiba (Curitiba, Paraná) e a Faculdade de Ciências Médicas da Universidade de Pernambuco (Recife, Pernambuco).

A escolha das capitais para o estudo, que ordenou por magnitude os 224 municípios do país com população acima de 100 mil habitantes, teve como base uma pesquisa realizada por Souza et al. ${ }^{19}$, para o Ministério da Saúde. Com base nela, foi elaborado um índice de violência que considerou o número de óbitos e as taxas de mortalidade por homicídio, acidente de transporte e suicídio no ano de 2000. Segundo esse índice, o Rio de Janeiro situou-se em segundo lugar; Recife, em terceiro; Brasília, em quinto; Manaus, em 13 e Curitiba, no 17o posto. Com exceção do Rio de Janeiro, todas as demais cidades têm as taxas de violência mais elevadas em sua região, segundo o referido indicador composto. No ano de 2000, as cinco cidades somaram 45.434 internações por causas externas, representando $6,5 \%$ das hospitalizações realizadas no país 19 .

A operacionalização dessa investigação foi construída em torno de quatro fases de trabalho, cada qual agregando diferentes ciclos de pesquisa (exploratório, trabalho de campo, análise e divulgação dos dados).

Na parte exploratória, entrevistas com 23 especialistas e gestores tiveram a finalidade de agregar informações, opiniões e relatos de experiências para subsidiar a elaboração dos instrumentos quantitativos e qualitativos do estudo. A análise dessa etapa foi acompanhada por jornadas de estudo de portarias de referência do Ministério da Saúde para o atendimento pré- hospitalar. Esses documentos tratam: (1) do incentivo financeiro para adequação da área física das Centrais de Regulação Médica de Urgência em estados, municípios e regiões de todo o território nacional - Portaria $n^{o}$. 1.828/GM, de 2 de setembro de 2004 4; (2) do grupo técnico para avaliar e recomendar estratégias de intervenção do Sistema Único de Saúde (SUS), para abordagem dos episódios de morte súbita - Portaria $n^{o}$. 2.420/GM, de 9 de novembro de 2004 4; (3) da Política Nacional de Atenção às Urgências, a ser implantada em todas as unidades federadas, respeitadas as competências das três esferas de gestão-Portaria $n^{\circ}$. 1.863/GM, de 29 de setembro de 2003 4; (4) do componente pré-hospitalar móvel da Política Nacional de Atenção às Urgências: SAMU-192 - Portaria $n^{o}$. 1.864/GM, de 29 de setembro de 2004 4; (5) do Comitê Gestor Nacional de Atenção às Urgências - Portaria $n^{\circ}$. 2.072, de 30 de outubro de $2003^{4}$, que faz várias modificações no sistema vigente, revogando a Portaria $n^{o}$. 814 , de $1^{\circ}$ de junho de 200120 e Portaria no ${ }^{\circ} .2 .048 /$ GM, de 5 de novembro de 2002 4. As diretrizes da Política Nacional de Redução da Morbimortalidade por Acidentes e Violências, no que concerne à sistematização, ampliação e consolidação do atendimento pré-hospitalar 2 , foi a referênciachave, apontando três focos para a atenção préhospitalar: (1) existência e padronização de veículos, materiais e rotinas, para os quais, nacional e internacionalmente, existem hoje suficientes conhecimento, orientação e planos já testados; (2) existência de integração e coordenação entre o atendimento pré-hospitalar e o hospitalar, objetivando à sobrevivência e ao conforto das vítimas; (3) mapeamento das áreas de risco para acidentes e violências, de modo a promover uma contra-referência visando a propostas e procedimentos de prevenção.

Na segunda fase, foi feito o mapeamento da rede. $\mathrm{O}$ instrumento construído, na forma de um questionário, objetivou a identificação das organizações governamentais que realizam atendimento às vítimas de acidentes e violências, contendo: (a) identificação da unidade; (b) caracterização das atividades de atendimento; (c) descrição da estrutura existente.

Na terceira fase, foram elaborados questionários específicos. No caso do atendimento préhospitalar, esse instrumento continha 26 questões distribuídas entre as seguintes temáticas: (1) identificação; (2) estrutura (número total de atendimentos por acidentes e violências, realizados nos anos de 2004 e primeiro semestre de 2005, equipamentos e pessoal disponíveis); (3) organização do serviço (mecanismos de transferência e transporte de pacientes; integração e suporte diagnóstico; articulação com a central de 
regulação; rotinas e protocolos de atendimento às vítimas de acidentes e violências; capacitação dos profissionais; indicadores do atendimento pré-hospitalar móvel; registro do atendimento).

Foram estudados os serviços de atendimento pré-hospitalar móvel (SAMU de Curitiba, Recife, Brasília, Rio de Janeiro; Grupamento de Socorro de Emergência - GSE do Rio de Janeiro; SOS e SAMU de Manaus) na sua história, processo de implantação, organização, recursos humanos, materiais e equipamentos. Os indicadores para medir a eficiência do sistema foram: (a) tempo médio de resposta das equipes de urgência: tempo transcorrido desde a hora da recepção do chamado na central de regulação até a chegada do SAMU/GSE ao lugar do sinistro; (b) tempo médio decorrido no local de ocorrência: tempo transcorrido desde a chegada do SAMU/GSE ao local do sinistro até a saída do cenário; (c) tempo médio de transporte até a unidade de referência; (d) tempo médio de resposta total: tempo transcorrido desde a hora da recepção do chamado pela central de regulação até a entrada do paciente no serviço hospitalar de referência.

Foram também elaborados roteiros de entrevistas semi-estruturadas destinadas aos gestores responsáveis pela coordenação do comitê gestor ou responsável pela Rede Pactuada de Atendimento às Emergências (ou equivalente) e ao coordenador ou diretor do serviço, identificado como responsável pelo resgate de vítimas de acidentes e violências nas capitais. Realizaram-se 16 entrevistas qualitativas: quatro em Manaus, três em Recife, três em Brasília, três no Rio de Janeiro e três em Curitiba. Ao término desse processo, houve um terceiro seminário interno para analisar o percurso da coleta de informações e preparar a fase de análise.

A quarta fase da pesquisa foi dedicada à análise crítica e ao aumento da qualidade dos dados e da validade das interpretações. Nesse movimento, todos os relatórios locais foram cuidadosamente analisados por profissionais de estatística, epidemiologia e ciências sociais do CLAVES e reenviados às equipes locais a fim de que se ajustassem as informações e sua forma de apresentação. A partir daí, os relatórios finais das cidades serviram de base para a construção da análise transversal, incluindo ainda, como matéria de consulta e exame, todos os acervos originais (banco de entrevistas e de dados das unidades).

Os questionários foram digitados em um banco de dados (Epidata; Epidata Association, Odense, Dinamarca) e, em seguida, submetidos a críticas. A análise quantitativa teve caráter exploratório, sendo usadas medidas estatísticas descritivas (freqüências simples e relativas) apli- cadas às variáveis em cada cidade. Na análise qualitativa, buscou-se interação entre conteúdos manifestos e inferência de conteúdos latentes, valendo-se dos depoimentos e das anotações de campo. As entrevistas foram processadas a partir de leitura temática e os trechos pertinentes foram codificados, identificando-se argumentos, significados e relatos de práticas relevantes, em face dos objetivos do trabalho. A seguir, foi realizado um exercício de triangulação das análises quantitativas e dos significados das relações e representações dos diferentes atores, na busca de uma visão abrangente e compreensiva do processo 21 .

\section{Resultados e discussão}

Sobre esse conjunto de ações do SAMU, realizouse uma análise descritiva e crítica, colocando-se esse subsistema no interior do sistema de urgência e emergência com ênfase em: transição entre formas anteriores; tipologia dos atendimentos; organização e interação entre serviços e gestão de recursos.

\section{Transição entre outras modalidades e efetivação do SAMU}

Esta análise se refere, principalmente, às mudanças que vêm ocorrendo nas funções exercidas tradicionalmente pelo Corpo de Bombeiros - que, na maioria dos estados, efetuava os serviços pré-hospitalares móveis - e à implantação do SAMU. A transição entre os modelos vem ocorrendo de forma desigual entre as capitais pesquisadas, embora a maioria dos gestores e profissionais envolvidos nos serviços pré-hospitalares considere o SAMU uma iniciativa bemvinda porque é capaz de agregar mais recursos e garantir maior cobertura. No entanto, especialmente onde o Corpo de Bombeiros já atuava com atendimento médico, avalia-se a perda de espaço político e de financiamento da corporação. Nesse momento de transição, destacam-se três realidades distintas.

Em Manaus, até janeiro de 2006, atuavam conjuntamente dois serviços: o SOS, de responsabilidade municipal, e o Resgate, realizado pelo Corpo de Bombeiros. Ambos não contavam com médicos na equipe e nem equipamentos adequados. Os dois serviços prestavam os primeiros cuidados, faziam imobilização de vítimas e as encaminhavam às unidades de saúde. Atendiam ainda, de forma complementar, pessoas de outros municípios com traumas graves.

Com a chegada do SAMU, coube ao Corpo de Bombeiros manauense apenas o resgate de 
vítimas nas chamadas “zonas quentes”, ou seja, onde e quando há risco de desmoronamento ou incêndio e em situações com pessoas presas a ferragens. Mesmo que a substituição da oferta anterior pela implantação do SAMU tenha sido bem aceita pelos gestores, a nova divisão do trabalho está ocorrendo com o ônus da insatisfação dos bombeiros. Eles se ressentem por não contarem com apoio e suporte financeiro do setor saúde ao programa já existente e pela desvalorização de tanta experiência acumulada.

Apesar das contradições, que estão sendo administradas, há grande expectativa de melhoria da qualidade do atendimento, de padronização de normas e procedimentos, de dotação, por parte do Ministério da Saúde, de equipamentos de resgate, de acordo com as características da região, carente, ainda, de unidades aéreas e fluviais para localidades de difícil acesso. A implantação do SAMU representa, para Manaus, a garantia de maior cobertura com expectativa de funcionamento de 14 unidades regionais, embora no momento apenas oito estejam em ação.

No Rio de Janeiro, o quadro de transição é mais conflituoso. Desde 1986, o Grupamento de Socorro de Emergência do Corpo de Bombeiros Militar do Estado do Rio de Janeiro (GSE/ CBMERJ) vinha sendo responsável pela assistência pré-hospitalar, que incluía equipes médicas para regulação e para atendimento. Durante a implementação do Programa Nacional de Enfrentamento de Emergências e Traumas, em 1992, o GSE colaborou, formando bombeiros para a atenção pré-hospitalar móvel em diversos estados.

Com a implantação do SAMU e o redimensionamento das funções entre instituições, a experiência dos bombeiros foi pouco considerada. Os gestores do GSE, quando entrevistados, teceram enfáticas críticas ao Ministério da Saúde por não ter envolvido a corporação na formulação e implantação do SAMU. Segundo eles, a interação entre os órgãos possibilitaria, além de maior eficiência, elevar consideravelmente o orçamento do GSE. A desconsideração foi interpretada por um dos gestores do GSE como uma clássica dificuldade que a área de saúde tem para agir de forma intersetorial, contrariando a imagem que o setor propala.

Na verdade, parece ter havido uma tímida tentativa de promover a integração, mas essa foi subjugada por entraves e questões de menor relevância, como obrigatoriedade do uso de logotipos, uniformes e equipamentos específicos. O certo é que o processo de capacitação dos profissionais que integrariam o SAMU no Rio de Janeiro foi realizado sem a participação do GSE, fato também considerado pelos entrevistados dessa corporação como pouco produtivo por ter focado conteúdos alheios à realidade fluminense. Certamente, o argumento considerado mais injusto no estremecimento das relações institucionais foi o que considerou improcedente o repasse de verbas da estrutura SAMU para os bombeiros.

Apesar dos conflitos, com a implantação do SAMU em 2005 foi estabelecido um acordo informal entre as partes, segundo o qual os bombeiros atenderiam apenas as urgências clínicas e domiciliares. Em outros termos, mesmo sem verba complementar, o GSE continua a ser o responsável pelo socorro pré-hospitalar móvel.

Na cidade de Curitiba, os gestores consideram que conseguiram uma boa articulação entre bombeiros e SAMU. O socorro era feito, desde 1990, pelo Sistema Integrado de Atendimento ao Trauma e Emergência (SIATE), gerenciado pelo Corpo de Bombeiros, que continuou responsável pela prestação de atendimentos às vítimas de acidentes e violências, agora de forma integrada ao Sistema de Atendimento Municipal às Urgências. Assim, o SIATE cuida das situações de acidentes e violências e o SAMU, de emergências clínicas. Ao ligar-se para a linha 193 do Corpo de Bombeiros, a central de comunicação transfere a ligação para a linha 192 (SAMU), onde uma central de regulação médica avalia a gravidade da situação e designa ambulância e equipe apropriada para atender ao chamado.

\section{Tipologia dos atendimentos e uso dos dados para planejamento}

Observando dados dos sistemas de resgate a vítimas de causas externas nos anos de 2004 e 2005 (Tabela 1), em cada capital estudada, concluise que o socorro para traumatizados no trânsito, transporte ou em demais acidentes constitui a maioria dos cuidados realizados. A atenção às lesões por agressões, em 2005, destacou-se nas cidades de Manaus, Rio de Janeiro e Curitiba.

Os gestores do programa no Rio de Janeiro e Recife assinalam que a notificação que realizam não segue a Classificação Internacional de Doenças, 10a revisão (CID-10). Contudo, representantes das cinco capitais afirmaram que realizam análise sistemática dos dados, tomando-os como base para o planejamento das ações. Uma vez questionados sobre com que nota avaliariam seus registros, os gestores atribuíram valores elevados (entre 7 e 10). No trabalho de campo, todavia, observaram-se contradições, pois os bancos de dados não estão articulados com os de outras instituições que prestam atendimento às vítimas. 
Número absoluto dos tipos de atendimento de pré-hospitalar móvel por acidentes e violência, realizados nas capitais brasileiras estudadas, nos anos de 2004 e 2005 *

\begin{tabular}{|c|c|c|c|c|c|c|c|c|c|c|}
\hline & \multicolumn{2}{|c|}{ Brasília } & \multicolumn{2}{|c|}{ Curitiba } & \multicolumn{2}{|c|}{ Manaus } & \multicolumn{2}{|c|}{ Recife } & \multicolumn{2}{|c|}{ Rio de Janeiro } \\
\hline & 2004 & 2005 & 2004 & 2005 & 2004 & 2005 & 2004 & 2005 & 2004 & 2005 \\
\hline Total de atendimentos & 514.349 & 537.282 & 824.022 & 429.144 & 964.892 & 680.358 & 363.514 & 213.794 & 1.547 .138 & 816.422 \\
\hline Atendimentos por agressão & 267 & 398 & 2.413 & 1.430 & 2.289 & 496 & 670 & 280 & 2.633 & 1.426 \\
\hline \multicolumn{11}{|l|}{ Atendimentos por lesão } \\
\hline autoprovocada & 139 & 129 & 41 & 26 & 20 & 0 & 143 & 66 & 554 & 326 \\
\hline \multicolumn{11}{|l|}{ Atendimentos por lesão decorrente } \\
\hline de intervenções legais & 47 & 70 & 0 & 0 & 0 & 0 & 0 & 0 & 0 & 0 \\
\hline \multicolumn{11}{|l|}{ Atendimentos por acidentes de } \\
\hline trânsito/transportes & 108 & 136 & 5.247 & 2.208 & 5.795 & 1.007 & 3.447 & 1.541 & 40.964 & 21.379 \\
\hline Atendimentos por quedas & 71 & 652 & 4.288 & 2.530 & 0 & 0 & 1.703 & 852 & 13.920 & 7.293 \\
\hline Atendimentos por demais acidentes & 118 & 473 & 390 & 134 & 0 & 81 & 396 & 185 & 3.278 & 1.732 \\
\hline
\end{tabular}

* No ano de 2005 só foram incluídos dados referentes ao primeiro semestre.

\section{Gestão de recursos do sistema}

Esse item trata dos equipamentos e medicamentos preconizados pela Portaria $n^{\circ}$. 2.048/ $G M{ }^{4}$ para um atendimento de qualidade. Os veículos apropriados são definidos de acordo com a Política Nacional de Atendimento às Urgências 4 . Ambulâncias (veículo terrestre, aéreo ou aquaviário que se destina exclusivamente a transporte de pacientes) podem ser do tipo A (próprias para remoções simples de caráter eletivo), B (adequadas ao suporte básico de vida para paciente com risco de morte em transporte inter-hospitalar e paciente do pré-hospitalar com risco de morte desconhecido), $\mathrm{C}$ (próprias para resgate, atendimento pré-hospitalar de vítimas de acidentes ou que estejam em locais de difícil acesso, com equipamento de salvamento) e D (visando ao suporte avançado de vida de paciente com alto risco e de transporte interhospitalar para os que necessitam de cuidados médicos intensivos e uso de equipamentos). É considerada do tipo $\mathrm{E}$ a aeronave de asa fixa ou rotativa para transporte inter-hospitalar e de resgate de paciente. Do tipo F é a embarcação para transporte em via marítima ou fluvial. Há, ainda, a previsão de meios de intervenção rápida, utilizando veículos leves para transporte de médicos com equipamentos para suporte avançado de vida e outros veículos adaptados para transporte de pacientes de baixo risco.

O SAMU preconiza os seguintes parâmetros demográficos para seus serviços: uma equipe de suporte básico de vida para cada 100/150 mil habitantes (um motorista, um auxiliar/técnico de enfermagem e uma ambulância tipo B); uma equipe de suporte avançado de vida para cada 400/450 mil habitantes (um motorista, um médico e um enfermeiro e uma ambulância tipo D); um médico regulador para cada central e um Núcleo de Educação em Urgência em cada capital 4.

A disponibilidade dos recursos previstos nas diversas portarias varia consideravelmente nas cinco capitais, embora em todas exista pelo menos um dos três tipos de ambulância preconizados: B, C ou D. Ao se examinar o que, efetivamente, existe em cada uma delas, verificam-se desigualdades e diferenças expressivas.

Em Manaus, por ocasião da pesquisa, não havia ambulâncias do tipo D e E, ainda que houvesse planejamento para a obtenção desses recursos. Em contrapartida, havia ambulâncias do tipo F. Não possuir unidades com suporte avançado de vida configura insegurança grave no cotidiano das operações. O SAMU de Recife não contava com ambulâncias do tipo $\mathrm{C}$ nem aeronaves. $\mathrm{O}$ subsistema dispunha de 15 ambulâncias e existia plano de expansão do atendimento, incorporando toda a região metropolitana. Esse plano previa aumento de cerca de um milhão e meio de habitantes na cobertura do sistema. Brasília somente possuía ambulâncias do tipo B e D, o que significa baixa diversidade de opções para cobrir as necessidades desse vasto complexo urbano. No Rio de Janeiro, o GSE/CBMERJ contava com 78 ambulâncias, sendo a metade com suporte avançado, presença de médicos, além de contar com todos os tipos previstos de ambulâncias. Curitiba possuía uma estrutura mais completa e coesa, 
reunindo vários serviços integrados de SIATE/ SAMU e suporte hospitalar. Isso representava um total de 64 ambulâncias (13 SIATE, 21 SAMU e 30 da central de ambulâncias). Dispunha dos tipos $\mathrm{B}, \mathrm{C}$ e D, menos aeronaves.

Foi pesquisada a disponibilidade de outros equipamentos e medicamentos preconizados pela Portaria $n^{\circ}$. 2.048/GM 4 e apenas o GSE do Rio de Janeiro preenchia todos os critérios. Apesar das deficiências constatadas, os gestores aferiram notas altas (entre 9 e 10) aos equipamentos e medicamentos disponíveis nos seus sistemas pré-hospitalares. Somente os de Brasília pontuaram esses quesitos com nota mais baixa (7). Um dos serviços de Manaus pontuou com nota $0 \mathrm{o}$ item medicamentos e atribuiu nota 9 aos equipamentos.

Pesquisou-se, também, a adequação dos recursos humanos à função. Os profissionais das cinco capitais têm formação apropriada e todas as equipes foram capacitadas em Advanced Trauma Life Support (ATLS). No entanto, há problemas em Brasília, pois o SAMU, em vez de contratar, desloca profissionais da rede de saúde. $\mathrm{O}$ gestor mencionou enfaticamente que existem deficiências na capacitação da equipe.

Em Manaus, os dois serviços existentes contam, juntos, com dois coordenadores, 27 enfermeiras, 164 auxiliares e 96 motoristas. Em Recife, existe perspectiva de mudança da sede do sistema para um espaço maior, expandindo o atendimento com regulação médica e aumentando o quadro de pessoal, que atualmente comporta um coordenador, 28 médicos, 17 enfermeiras, 84 auxiliares de enfermagem e 70 motoristas. $\mathrm{O}$ GSE do Rio de Janeiro tem a maior equipe dentre todas as capitais analisadas: 14 coordenadores, 221 médicos, 4 enfermeiras, 492 auxiliares de enfermagem, 1 assistente social. Já o SAMU de Curitiba opera com 7 coordenadores, 23 médicos na central de regulação, 47 médicos nas unidades avançadas de suporte, 72 auxiliares de enfermagem, 28 enfermeiros e 68 motoristas.

A avaliação dos gestores quanto ao número e qualificação dos profissionais disponíveis para o atendimento recebeu notas mais críticas em Brasília (6 e 5 respectivamente) e em Manaus (nota 6 para a qualificação dos socorristas).

A presteza no socorro é um dos itens fundamentais para a atenção pré-hospitalar. Na Tabela 2, apresentam-se os tempos médios gastos nos serviços móveis, por cada capital, e, considerando o tempo de resposta total como um dos indicadores de qualidade da atenção, é possível trabalhar com algumas hipóteses.

As informações da Tabela 2 indicam que Manaus aponta o tempo menor para resposta total, entretanto esse dado é fruto de estimativa e provavelmente indica pouca precisão. Recife apresenta uma medida intermediária. Rio de Janeiro e Curitiba têm comportamentos opostos. Esta capital assinala um dos menores tempos, sendo favorecida pelo trânsito mais ordenado e por uma boa articulação na interação da unidade móvel com as unidades pré-hospitalares fixas e hospitais - fato esperado em virtude do modelo integrado existente na cidade. Por sua vez, o GSE do Rio de Janeiro gasta poucos minutos para chegar ao local onde se encontra a vítima, porém despende muito tempo até a unidade fixa de atendimento. Os gestores comentam que isso se deve à precária articulação entre o pré-hospitalar móvel e a recepção do paciente nas unidades da rede pública.

“Às vezes eu estou aqui; pego o doente e aqui tem um hospital. Só que não posso levar pra cá porque esse hospital não tem capacidade de receber o doente; porque está faltando o Neuro. Tenho que atravessar a cidade para outro lugar. Os problemas maiores encontrados pela central da regulação, é que eu não tenho hospital de referência, então isso aqui é um verdadeiro inferno brasileiro.

Tempos médios (em minutos) do atendimento pré-hospitalar, segundo as capitais brasileiras estudadas, no primeiro semestre de 2005.

\begin{tabular}{|c|c|c|c|c|c|}
\hline & Manaus & Recife & Brasília & Rio de Janeiro & Curitiba \\
\hline Resposta das equipes de urgência & 2 & 15 & 10 & 9 & 10 \\
\hline Tempo médio decorrido no local de ocorrência & 15 & 20 & - & 13 & 20 \\
\hline Tempo médio de transporte até a unidade de referência & 5 & 10 & 20 * & 20 & 6 \\
\hline Espera para o primeiro atendimento & - & 15 & - & 9 & 8 \\
\hline Resposta total & 20 & 45 & - & 66 & 35 \\
\hline
\end{tabular}

* Quando se trata de um regaste em Brasília, o tempo estimado foi de 20 minutos; quando o resgate era em alguma cidade satélite, o tempo foi de 30 minutos. 
Tenho tomógrafo, mas não tenho operador; tenho tomógrafo e operador, mas não tenho filme. O dia que tenho os três, falta ar-condicionado. A gente tem uma falta muito grande de especialista e de vaga, porque a emergência não é emergência: todo mundo procura, porque sabe que algum médico vai atender" (gestor 2 pré-hospitalar - Rio de Janeiro).

Para minimizar o quadro descrito, diretores e chefes de emergência dos hospitais públicos e representantes do Corpo de Bombeiros se reúnem mensalmente numa Câmara Técnica do Conselho Regional de Medicina do Rio de Janeiro, na busca de articulação entre os setores préhospitalar e de emergência. Esses acordos, contudo, constituem compromissos informais, sem vincular responsabilidades às instâncias gestoras formais. A precária articulação entre esses dois setores é agravada pela baixa disponibilidade de vagas nos hospitais, problema que atinge também várias capitais. A demanda ambulatorial de pessoas com doenças crônicas que buscam os serviços de urgência e o número insuficiente de profissionais foram problemas pontuados pelos gestores de todas as capitais estudadas. Estudos de base etnográfica vêm revelando que a população possui critérios peculiares, a partir de valores e referências, para denominar o que seja uma urgência, e esses parâmetros nem sempre se assemelham aos da biomedicina 11,22 .

Apesar de os gestores do subsistema pré-hospitalar móvel afirmarem que existe boa articulação com a rede hospitalar através de central de regulação ou da rede pactuada de serviços (no caso do Rio de Janeiro, esta é operada pelos bombeiros), eles aferiram notas mais baixas para essa interação. Os do Rio de Janeiro, Brasília e Curitiba revelaram maior insatisfação, atribuindo-lhe nota 6. Ao contrário, os de Manaus e Recife valorizaram essas relações com a nota 9. Todos os serviços pré-hospitalares das capitais, segundo os gestores, apresentam rotinas e protocolos para o atendimento às vítimas de acidentes e violências.

Os gestores realçaram outras dificuldades para que o subsistema pré-hospitalar móvel opere com eficiência: (a) chamadas feitas por pessoas portadoras de doenças crônicas que lançam mão desse recurso como alternativa às dificuldades de locomoção, fator de grande relevância em Recife; (b) dificuldade de provimento rápido da manutenção de ambulâncias, o que é mais grave em Manaus; (c) trotes, que correspondem a cerca de $60 \%$ das ligações feitas ao SAMU de Brasília, sendo também problema relevante em Curitiba.

\section{Conclusões}

Nesta conclusão ressaltam-se alguns pontos que sobressaíram na pesquisa empírica:

- Na continuidade do processo de transição, é preciso que a parceria entre Corpo de Bombeiros e SAMU continue a ser fortalecida, especialmente no Rio de Janeiro, onde se observa acirramento das divergências. A competência e a experiência do Corpo de Bombeiros no Rio de Janeiro é um patrimônio local reconhecido em todo o país que deveria ser valorizado, inclusive por meio de suporte financeiro.

- A implantação do SAMU nas capitais estudadas vem sendo considerada muito positiva, sobretudo quando se considera que o resgate móvel não dispunha de centrais médicas de regulação e de atendimento médico nas ambulâncias, como é o caso de Manaus. Apesar de alguns problemas específicos e compreensíveis nos processos de transição, nas cinco capitais a implantação do SAMU vem representando benefício para a população.

- A articulação do pré-hospitalar móvel com as demais unidades de saúde por intermédio da central de regulação também é um ponto positivo nas capitais. Todavia, no Rio de Janeiro, os gestores assinalam que existem sérios entraves para a obtenção de vagas nos hospitais da rede. Essa desconexão interfere negativamente na rapidez do atendimento prestado pelo GSE que, apesar de sua eficiência, perde tempo precioso até a entrega do paciente nas unidades de saúde. Decisões tomadas pelos gestores para solucionar o problema vêm servindo para pactuar informalmente a oferta de leitos e trocar informações sobre a disponibilidade dos serviços, porém os acordos não instituem compromissos e responsabilidades formais entre secretarias, programas e hospitais. Em resumo, a falta de vagas nos hospitais agrava a baixa articulação entre os setores de atendimento pré-hospitalar e hospitalar, o que também foi assinalado pelos gestores de Brasília e Curitiba. Esse é um problema estrutural do SUS que precisa de alternativas viáveis, sobretudo nos serviços de atenção às vítimas de violências e acidentes, em que o tempo é um dos fatores mais relevantes para salvação de vidas.

- É importante dar ênfase às informações com finalidade de planejamento. Tendo em vista que traumas graves provocados por acidentes e violências são os principais tipos de agravos que exigem atenção específica, é de se esperar que todas as capitais possuam, pelo menos, todos os tipos de ambulância previstos para a estruturação do SAMU. Isso não vem ocorrendo, a não ser no Rio de Janeiro. Igualmente, o supri- 
mento de equipamentos e medicamentos para os primeiros socorros constitui exigência de altíssima relevância para esse tipo de serviço, e a sua escassez ou falta mereceu crítica de muitos gestores.

A formação de recursos humanos especializados é um ponto positivo em quatro das cinco capitais estudadas. Merece especial atenção o caso de Brasília, onde este ponto está descuidado. É necessário que a Secretaria de Saúde de Brasília forme seu quadro próprio para o SAMU, o que deveria ser monitorado e exigido pelo Ministério da Saúde.

\section{Resumo}

Apresentamos descrição e análise da implantação do sistema de atendimento pré-hospitalar móvel (Serviço de Atendimento Móvel de Urgência - SAMU). O texto é parte de uma pesquisa denominada Análise Diagnóstica de Implantação da Política Nacional de Redução de Acidentes e Violências. Estudamos a história recente da implantação, organização, recursos humanos, materiais e equipamentos do SAMU em cinco capitais (Curitiba - Paraná; Recife - Pernambuco; Brasília - Distrito Federal; Manaus - Amazonas; Rio de Janeiro) que apresentam elevadas taxas de morbimortalidade por causas externas. Trabalhamos em quatro fases, cada qual agregando ciclos exploratórios, de trabalho de campo e de análise, triangulando dados quantitativos e qualitativos. Os resultados mostram que a implantação do SAMU constitui, hoje, um avanço do setor saúde e da sociedade. É preciso ainda completar a implantação de várias portarias quanto a veículos, pessoal e equipamentos; intensificar a articulação do pré-hospitalar móvel com as unidades de saúde; enfatizar informações geradas nesse subsistema visando ao melhor planejamento das ações; manter e promover a alta qualificação dos profissionais do $S A M U$. Este serviço veio oficializar, padronizar e regular um subsistema fundamental para salvar vidas.

Assistência Pré-Hospitalar; Emergências; Serviços PréHospitalares
Para a maioria dos gestores e profissionais que atuam na oferta de serviços pré-hospitalares às vítimas de lesões e traumas por acidentes e violências, o SAMU é hoje um bem que o setor saúde oferece à sociedade brasileira. Esse tipo de serviço veio oficializar, padronizar e regular um subsistema fundamental para salvar vidas, tendo já sido comprovada sua eficácia em vários países do mundo. Portanto, é preciso que os administradores do SUS invistam na sua continuidade, no aperfeiçoamento de sua implantação e implementação e no seu monitoramento, buscando excelência e integração com todo o sistema de urgência e emergência.

\section{Colaboradores}

M. C. S. Minayo foi responsável pela redação do artigo. S. F. Deslandes foi responsável pela apresentação dos dados, tabelas e trabalho de campo. 


\section{Referências}

1. Ministério da Saúde. Decreto no ${ }^{\circ}$. 5.055. Institui o Serviço de Atendimento Móvel de Urgência SAMU, em municípios e regiões do território nacional, e dá outras providências. Diário Oficial da União 2004; 27 abr.

2. Ministério da Saúde. Portaria $n^{\circ}$. 737, de 16 de maio de 2001. Política Nacional de Redução de Morbimortalidade por Acidentes e Violências. Diário Oficial da União 2001; 18 mai.

3. Minayo MCS, Deslandes SF, organizadoras. Análise diagnóstica: da Política Nacional de Redução de Acidentes e Violências. Rio de Janeiro: Editora Fiocruz; 2007.

4. Ministério da Saúde. Política Nacional de Atenção às Urgências. Brasília: Ministério da Saúde; 2004.

5. Hospital and prehospital resources for optimal care of the injured patient. Committee on Trauma of the American College of Surgeons. Bull Am Coll Surg 1986; 71:4-23.

6. Pepe PE, Wyatt CH, Bickell WH, Bailey ML, Mattox KL. The relationship between total prehospital time and outcome in hypotensive victims of penetrating injuries. Ann Emerg Med 1987; 16:293-7.

7. Whitaker IY, Gutièrrez MGR, Koizumi MS. Gravidade do trauma avaliada na fase pré-hospitalar. Rev Assoc Med Bras 1998; 44:111-9.

8. Trunkey DD. Trauma. Accidental and intentional injuries account for more years of life lost in the U.S. than cancer and heart disease. Among the prescribed remedies are improved preventive efforts, speedier surgery and further research. Sci Am 1983; 249:28-35.

9. Champion HR, Copes WS, Sacco WJ, Lawnick MM, Keast SL, Bain Jr. LW, et al. The Major Trauma Outcome Study: establishing national norms for trauma care. J Trauma 1990; 30:1356-65.

10. Albuquerque VS, Minayo MCS. Atendimento préhospitalar de emergência: referenciais técnicos, gestão dos serviços e atuação profissional. Cad Saúde Pública; submetido.
11. Deslandes SF. Frágeis deuses: profissionais da emergência entre os danos da violência e a recriação da vida. Rio de Janeiro: Editora Fiocruz; 2002.

12. Lechleuthner A, Emerman C, Dauber A, Bouillon B, Kubincanek JA. Evolution of rescue systems: a comparison between Cologne and Cleveland. Prehosp Disaster Med 1994; 9:193-7.

13. Hayashi Y, Hirade A, Morita, H. An analysis of time factors in out-of-hospital cardiac arrest in Osaka Prefecture. Resuscitation 2002; 53:121-5.

14. Rainer TH, Houlihan KP, Robertson CE, Beard D, Henry JM, Gordon MW. An evaluation of paramedic activities in prehospital trauma care. Injury 1997; 28:623-7.

15. Nikkanen HE, Pouges C, Jacobs LM. Emergency medicine in France. Ann Emerg Med 1998; 31: 116-20.

16. Denzin NK. The research act. Chicago: Aldine Publishing Company; 1973.

17. Minayo MCS, Sanches O. Quantitativo-qualitativo: oposição ou complementaridade? Cad Saúde Pública 1993; 9:237-48.

18. Minayo MCS, Souza ER, Assis, SG, organizadores. Avaliação por triangulação de métodos: abordagem de programas sociais. Rio de Janeiro: Editora Fiocruz; 2005.

19. Souza ER, Minayo MCS, Silva CMFP, Reis AC, Malaquias JV, Veiga JPC, et al. Análise temporal da mortalidade por causas externas no Brasil: décadas de 80 e 90. In: Minayo MCS, Souza ER, organizadores. Violência sob o olhar da saúde: a infrapolítica da contemporaneidade brasileira. Rio de Janeiro: Editora Fiocruz; 2003. p. 83-107.

20. Ministério da Saúde. Portaria no. 814/GM, de 1o de junho de 2001. Estabelecer na forma Anexo I, desta Portaria, o conceito geral, os princípios e as diretrizes da Regulação Médica das Urgências. Diário Oficial da União 2001; 1 jun.

21. Minayo MCS. O desafio do conhecimento: pesquisa qualitativa em saúde. 9ạ Ed. São Paulo: Editora Hucitec; 2006.

22. Giglio-Jacquemot AG. Urgências e emergências em saúde: perspectivas de profissionais e usuários. Rio de Janeiro: Editora Fiocruz; 2005.

Recebido em 13/Set/2007

Aprovado em 26/Dez/2007 